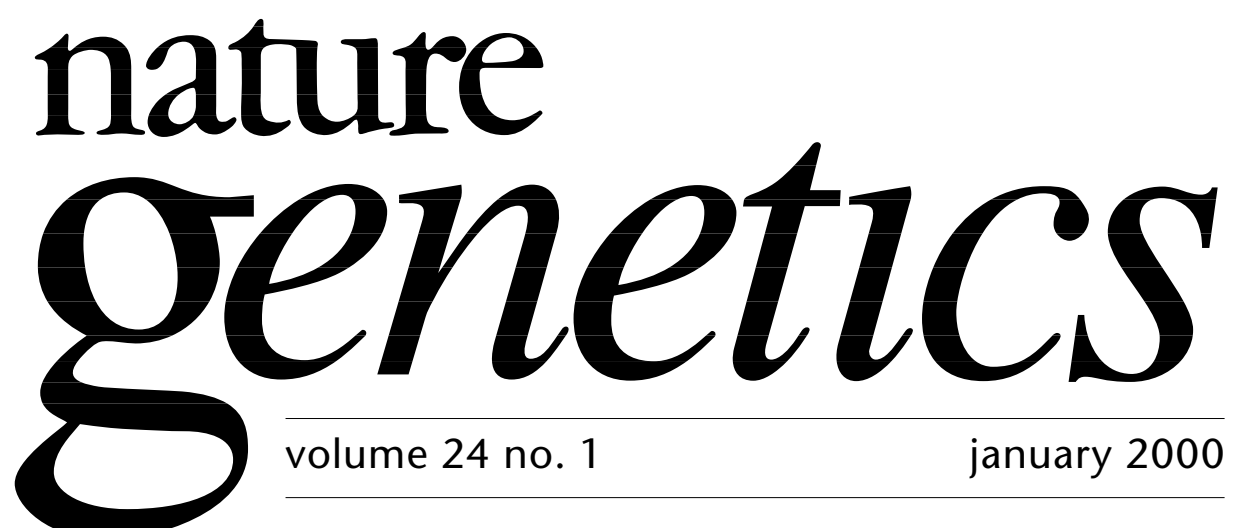

\title{
Genetics on-line
}

As genetic information begins to influence mainstream medical practice, the need to educate both practitioners and the public on its implications for healthcare will escalate. In her presidential address at the 1999 American Society of Human Genetics meeting, Uta Francke emphasized that public awareness and understanding of genetics could be facilitated by web sites that provide accurate and timely genetic information. But existing web-based resources seem ill equipped for this task.

The paucity of web sites that provide general information on genetics is in stark contrast to more than 17,000 web sites dedicated to healthcare information. Although some healthcare sites mention genetics, the information, according to Joseph McInerney of the Foundation for Genetic Education and Counseling (FGEC), is limited and often outdated. The sites that are dedicated exclusively to genetics, such as GeneClinics and the Alliance of Genetic Support Groups, are predominantly of a specialist nature, catering to clinical geneticists, genetic counsellors, and patients with rare monogenic disorders. Why are there so few web sites that provide lay information about genetics? Despite the advances in genetic research, the health implications are restricted so far to a relatively small portion of the population. Clinical genetics currently occupies a narrow niche of medicine, with roughly 1,000 board-certified clinical geneticists and 1,400 board-certified genetic counsellors in the United States. In the United Kingdom, there are approximately 100 clinical geneticists and 160 qualified counsellors.

Several groups hope to bridge this gap by developing new web-based resources to inform the public about the impact of genetics on general healthcare. For example, the FGEC, supported in part by Genset, is developing a framework to educate consumers and general practitioners about the genetics of common diseases and human variation. Celera is sponsoring the creation of an on-line magazine, tentatively called GeneWeek, to provide the lay reader with news updates on research and policy related to genomics. GeneSage, a commercial web site, aims to provide consumers and primary physicians with a comprehensive overview of genetics and will host an electronic magazine, offer distance learning for medical and nursing practitioners, and broadcast on-line lectures. The site is being developed with funds from private investors and according to Philip Reilly (Shriver Center), one of GeneSage's cofounders, future revenue is expected to come from advertisers.

Information can be an asset but also a liability, and the anticipated proliferation of web sites providing genetic information raises issues of accuracy, comprehensiveness and, given the pace of genetic research, frequency with which information is updated. These and other concerns were aired at a meeting convened by The 
National Human Genome Research Institute (NHGRI) and the Office of Rare Diseases last August, which brought together 28 not-for-profit organizations that currently provide, or intend to supply, genetic information over the Internet. The group, called Genetic Resources on the Web (GROW), plans to establish the minimal standards for site quality and a mechanism for evaluating genetics web sites. This is reminiscent of an initiative by Health Internet Ethics (Hi-Ethics), an alliance of medical web sites including Medscape, drkoop.com and WebMD, to establish an ethical code of conduct to guarantee reliable and trustworthy health information.

Given the magnitude of the Internet, it is difficult to envisage a 'watchdog' patrolling web sites that provide genetic information. But a group, such as GROW, that could evaluate and put a 'stamp of approval' on web sites that fulfil standards set by the genetics community would offer the public a means of identifying reliable sites. Alternatively, assessment and endorsement could be undertaken by an established international body, such as the Human Genome Organization,

Some genetic web sites for healthcare providers and the public

- National Coalition for Health Professional Education in Genetics http://www.nchpeg.org

- A World of Genetic Societies http://www.faseb.org/genetics

- Foundation for Genetic Education and Counseling http://www.genetic-medicine.org

- GeneClinics http://www.geneclinics.org

- Alliance of Genetic Support Groups http://www.geneticalliance.org

- Genetic Interest Group (GIG) http://www.gig.org.uk

- Access Excellence http://www.accessexcellence.com

- University of Kansas Medical Center, Genetics Education Center http://www.kumc.edu/gec

- The DNA Learning Center http://vector.cshl.org

- Education outreach programs: http://genetics-education.mbt.washington.edu/database which has programs dedicated to ethical and educational issues relevant to genetics. Regardless of the organization, the effort should be international, encompass both English and non-English sites, and include for-profit web sites. Such an endeavour would require substantial administrative and financial support, but should be encouraged by the genetics research community if it is to have a say in the quality and content of web-based information that conveys the output of its research to the public.

Alan Guttmacher, who coordinates the NHGRI's involvement in GROW, emphasizes that the aim of the group is not solely to review genetics web sites, but also to provide a constructive and supportive forum for discussing technical problems and the content of developing sites. To ensure that they reach the intended audience, web sites must be 'visible' to mainstream web directories and search engines. GROW is investigating ways to ensure its member sites are discovered by external search engines and also plans to develop the capability to simultaneously search across all members' sites. Guttmacher hopes that this could lead to the creation of a GROW homepage that would serve as a portal through which both health professionals and the public could access reliable information from a range of sites.

People that suffer from, or are at risk of developing, a genetic disorder usually are informed of their condition by their physician. But with the proliferation of webbased services that provide information on inherited susceptibility, there is a danger that individuals may attempt self-diagnosis based on information gleaned from the Internet without being appropriately counselled on relative risks, potential treatments or preventative measures. If genetics web sites are to benefit healthcare, it is imperative not only that the information be of high quality, but also that steps are taken to ensure that consumers who would benefit from counselling are directed to appropriate professional support. Furthermore, it is critical that medical practitioners keep abreast of developments in genetics and, once again, webbased resources could address this need.

It is no surprise that many organizations, particularly commercial groups, are sponsoring web sites to educate the public about genetics. Efforts to market 'individualized' medicines based on genetic profiles are likely to have a greater chance of success if potential consumers are primed-a lesson learned from the backlash against genetically modified foods. Whereas a healthy degree of competition between not-for-profit and commercial efforts is desirable, it is clearly in the best interests of everyone if web sites providing the public with information on genetics meet a common set of standards.

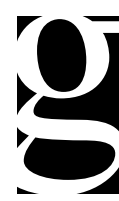

